\title{
Early surgery did not reduce 6-year mortality in patients with small abdominal aortic aneurysms
}

The UK Small Aneurysm Trial Participants. Mortality results for randomised controlled trial of early elective surgery or ultasonographic sureillance for small abdominal antic aneurysms. Lancet. 1998 Nov 21 ; $352: 1649-55$

\section{Question}

In patients with small abdominal aortic aneurysms, does early elective open surgical repair reduce mortality better than regularultrasonographic surveillance of aortic diameter?

\section{Design}

Randomized, unblinded, controlled trial with up to 7 years of follow-up (mean $4.6 \mathrm{y})$.

\section{Setting}

93 hospitals in the United Kingdom.

\section{Patients}

1090 patients who were 60 to 76 years of age (mean age $69 \mathrm{y}, 83 \%$ men); were fit for elective surgery; and had symp tomless, infrarenal, abdominal aortic aneurysms 4.0 to $5.5 \mathrm{~cm}$ in diameter (mean diameter $4.6 \mathrm{~cm}$ ). Exclusion criteria included being unfit for elective surgery and having a symptomatic aneurysm. Follow-up was complete.

\section{Intervention}

Patients were allocated to early surgery $(n=563)$ or ultrasonographic surveillance of the anearysm diameter $(n=527)$. Surgery was done according to normal local procedures. In the surveillance group, patients with aneurysms 4.0 to $4.9 \mathrm{~cm}$ were seen every 6 months, and those with aneurysms 5.0 to $5.5 \mathrm{~cm}$ were seen every 3 months; elective surgical repair was recommended to patients if the aneurysm diameter was $>5.5 \mathrm{~cm}$, the growth rate was $>1 \mathrm{~cm} / \mathrm{y}$, the aneurysm became tender, or iliac or thoracic repair was needed.

\section{Main outcome measure \\ Death from all causes.}

\section{Main results}

Analysis was by intention to treat. In the surgery group, 517 of 563 patients (92\%) had elective aneurysm repair with a prosthetic inlay graft; 452 of these patients $(87 \%)$ had surgery $\leq 5$ months after randomization (median time to surgery $1.8 \mathrm{mo}) .38(7 \%)$ patients in the surveillance group had surgery contrary to study protocol. By 6 years, mortality rates did not differ between the groups (hazard ratio $0.94,95 \%$ CI 0.75 to 1.17 , $P=0.56$ ) (Table). Results were similar after adjustment for baseline factors and source of patientreferral. The stady had $80 \%$ power to detect a $9 \%$ difference at 5 years of follow-up.

\section{Conclusion}

Early, prophylactic, elective surgery did not reduce mortality compared with ultrasonographic surveillance of aortic diameter in patients with small, asymptomatic, abdominal aortic aneurysms.

Sources of funding; Medical Researd Council and British Heant Foundation.

For correspondlence: Professor 7.T. Powell, Department of Vacular Surgery, Inperial Callege School of Meditine, Charing Cross Hoxpital, London W6 8RF, England, UK. IAX 44-181-846-7330.

Absuact and Commentary also published in ACP 7ounal Chl. 1999;130:73.

Early elective surgery vs ultrasonographic surveillance of aortic diameter for small abdominal aortic anetrysms*

\begin{tabular}{lcccc}
\hline Outcome & Surgery & Surveillance & RRR $(95 \% \mathrm{CD})$ & NNT \\
\hline Death at $6 \mathrm{y}$ & $28.2 \%$ & $28.5 \%$ & $0.8 \%(-19.9$ to 17.8$)$ & Not significant \\
\hline
\end{tabular}

*Abbreviations defined in Glossary; RRR, NNT, and Cl calculated from data in article.

\section{Commentary (continued from page 88)}

health care system to another. Unfortunately, few data about the amount of resources used by patients in the study (e.g., mean number of ultrasonograms and mean days in the hospital) were provided, which makes it difficult to compare treatments across regions. Further, patient-borne costs were not inchided, al though these are unlikely to have been a major factor in the analysis.

The finding that patients in the surgery group had less bodily pain and more improved heatth perceptions than patients in the surveiliance group is intriguing. One can hypothesize that patients in the surveillance group were more anxious that their anevrysm might rupture and, thus, had poorer health perceptions. Now that patients can be told that ultrasonographic surveillance leads to outcomes similar to those of early surgery, one wonders whether their heald perceptions would also be similar. It is not clear why bodily pain should be improved in the surgery group.

It is likely that some patients will always prefer to have their aneurysm repaired earlier rather than later, whereas others will be comfortable to have their aneurysm followed for some time. Now that this excel lent study has provided data about the outcomes of immediate surgery compared with: ultrasonographic surveillance, the time seems right to develop a decision aid to give patients detailed information about the choices available to them and the consequences of those choices $(5,6)$. This will allow patients to participate fully in the decision about the timing of surgery and to make the choice that is right for them.
Andreas Laupacis, MD, MSi Loeb Heart Researb Unit Ottava, Ontario, Canada

Reforences

1. Lederle FA, Wilson SE, Johuson GR, et al. J Vasc Surg. 1994;20:296-303.

2. May $\}$, White GH, $Y_{u} W$, et al. J Vasc Surg. 1998:27:213-20; discussion 220-1.

3. May J, White GH, Waugh $R$, et al. J Vase Surg. 1999:29:32-7; discussion 38-9.

4. Zarins CK, White RA, Schwarten D, et al. I Vasc Surg. 1999;29:292-308.

5. O'Connor AM, Tugwell $P$, Wells GA, et al Patient Educ Couns. 1998;33:267-79.

6. Barty MI, Cherkin DC, Chang $Y$, Fowler FJ, Skates S. DMCO. 1997;1:5-14. 